\title{
CUTANEOUS ASPERGILLOSIS IN A BOY WITH ACUTE LEUKEMIA: THE LESSONS FROM A "SIMPLE" CASE
}

\author{
Milena Bozhkova ${ }^{1}$, Temenuga Stoeva $^{1}$, Bonka Marinska ${ }^{2}$, Valeria Kaleva ${ }^{2}$, \\ Deyan Dzhenkov ${ }^{3}$, Igor Resnik ${ }^{4}$ \\ ${ }^{1}$ Department of Microbiology and Virology, Faculty of Medicine, \\ Medical University of Varna \\ ${ }^{2}$ Department of Pediatrics, Faculty of Medicine, Medical University of Varna \\ ${ }^{3}$ Department of Clinical Pathology, Faculty of Medicine, Medical University of Varna \\ ${ }^{4}$ Department of Medical Genetics, Faculty of Medicine, Medical University of Varna
}

\begin{abstract}
Fungal infections are among the most serious infectious complications in immunosuppressed patients. Primary cutaneous aspergillosis is a relatively rare manifestation and is usually described in patients with underlying malignant hematological diseases, AIDS, allogenic stem cell transplantation, solid organ transplantation, etc. We present a clinical case of a 12-year-old boy with acute lymphoblastic leukemia initially diagnosed as primary cutaneous aspergillosis caused by Aspergillus flavus. The retrospective analysis demonstrates that a small solitary pulmonary nodule could be a possible portal of entry. This means that we could consider the disease as an early stage invasive aspergillosis, which is successfully treated with voriconazole.
\end{abstract}

Keywords: cutaneous aspergillosis, invasive aspergillosis, Aspergillus flavus, voriconazole

\section{INTRODUCTION}

Fungi belonging to the genus Aspergillus are typical opportunistic pathogens. Due to airborne contamination the lungs and sinuses are the most common primary sites of infection, although Aspergillus can disseminate to distant organs and manifest as invasive aspergillosis (IA). Primary cutaneous le-

Address for correspondence:

Milena Bozhkova

Department of Microbiology and Virusology

Faculty of Medicine

Medical University of Varna

55 Marin Drinov St

9002 Varna

e-mail:drbojkovam@abv.bg

Received: April 18, 2018

Accepted: June 18, 2018 sions as a result of direct implantation of Aspergillus spores can occur in some cases and are usually developed after skin barrier disruption (1). Primary cutaneous aspergillosis (PCA) in immunocompromised patients has the potential to progress to systemic infection and is frequently lethal $(1,2)$. An adequate and rapid microbiological diagnosis is of essential importance for the prompt therapy of these patients. Here we present a clinical case of a 12-year-old boy initially diagnosed with PCA, successfully treated with voriconazole. Later the disease was defined as an early stage of IA.

\section{CASE DESCRIPTION}

A 12-year-old patient presented with a lowgrade fever, weakness, bone and joint pain, lymphadenopathy, hepatosplenomegaly and WBC 178 $\mathrm{x} 10^{9}$ per liter. Bone marrow aspirate smears revealed $100 \%$ infiltration with lymphoblasts. Diag- 
Milena Bozhkova, Temenuga Stoeva, Bonka Marinska et al.

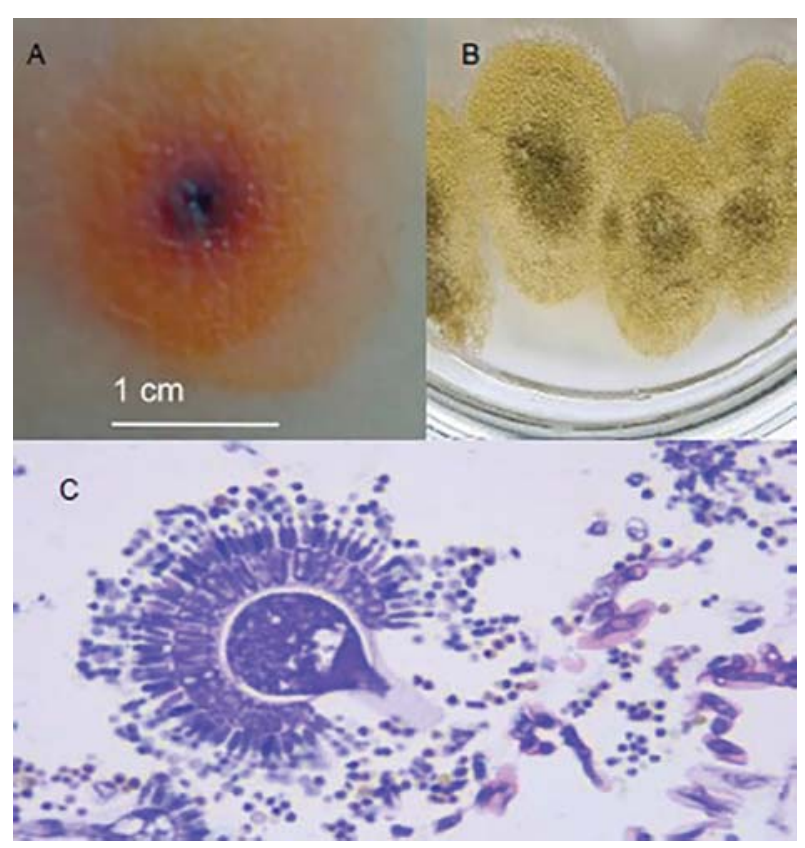

Fig. 1. Cutaneous aspergillosis. A. The initial presentation of described in text skin lesion. B. Colonies of Aspergillus flavus after 48 hours of incubation on Sabouraud agar. C. Microphotography of the skin biopsy sample demonstrates necrotic zones, with numerous branched hyphae and conidiospores suggestive of Aspergillus species.

nosis of high-risk T-cell acute lymphoblastic anemia with central nervous system (CNS) involvement was made and course of chemotherapy according to the phase 1 of ALL IC BFM 2009 Protocol was started.
The response was poor on D8 and remission was not achieved at the end of the induction on D33.

While the patient was neutropenic, a small skin lesion was noticed on the back side of the patient's right hip. The lesion progressed into weakly painful ulcerative papule with a size of approximately $8 \mathrm{~mm}$ with central necrosis, surrounded by a zone of local hyperemia (Fig. 1A). A skin biopsy sample was collected and cultured on Sabouraud agar at $30^{\circ} \mathrm{C}$ and after 48 hours mold colonies with yellow-green pigmentation were found (Fig. 1B). Microscopic examination of colonies revealed the typical morphological characteristics of Aspergillus flavus. Histopathological examination of the skin biopsy sample demonstrated adipose tissue with inflammatory cells and necrotic zones, numerous branched dichotomous at $45^{\circ}$ angles septate hyphae and conidiospores suggestive for Aspergillus spp. (Fig. 1C).

Computed tomography (CT) scanning of the chest was done twice and revealed normal lung parenchyma with a single small nodular lesion and no other typical for invasive pulmonary aspergillosis (IPA) signs (cavitation, halo sign, etc.) (Fig. 2). Serum levels of galactomannan were tested twice and both results were negative.

Antifungal therapy with voriconazole was initiated with $600 \mathrm{mg} / \mathrm{d}$ on the first day, and then 400 $\mathrm{mg} / \mathrm{d}$. The second lung CT performed after 6 weeks
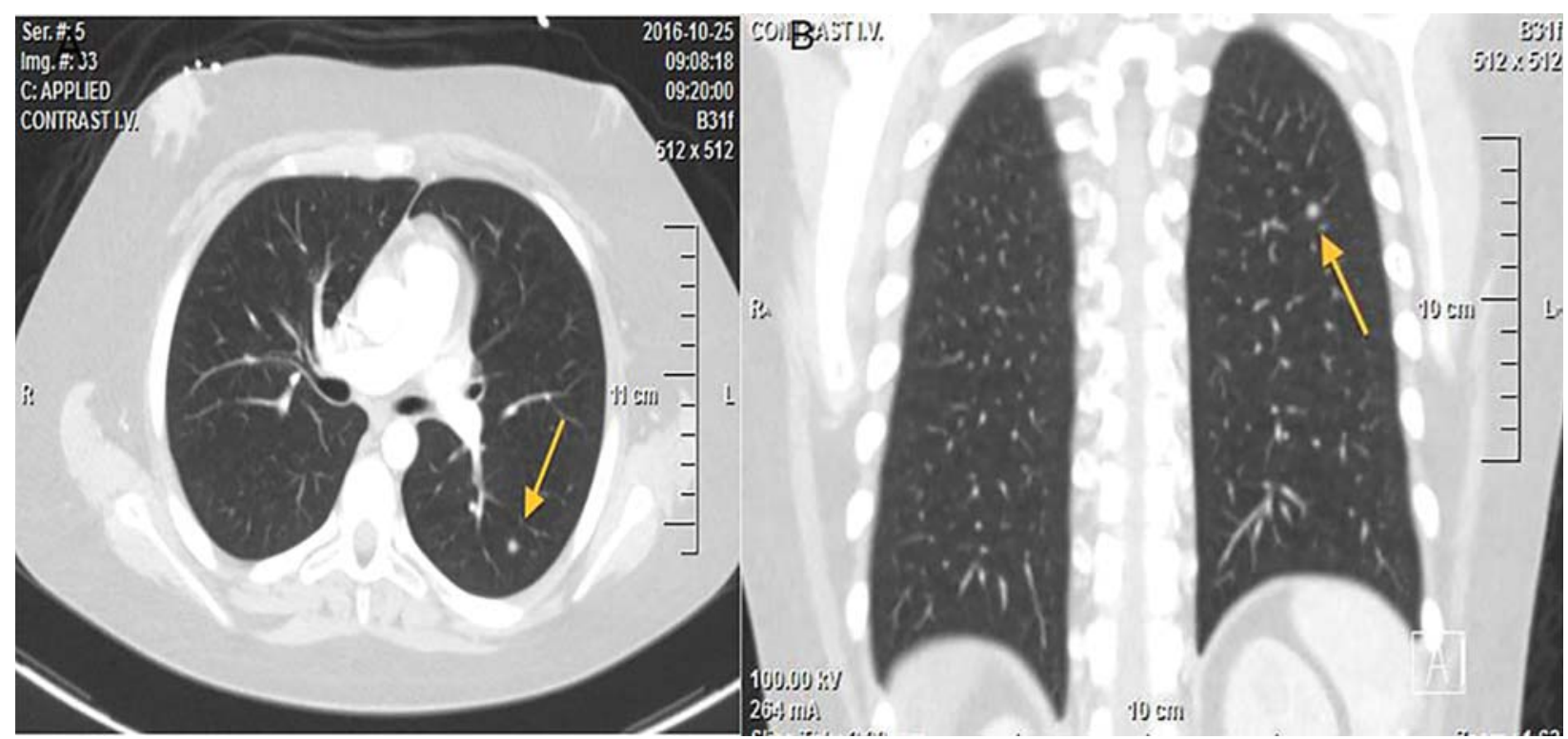

Fig. 2. CT scan before the start of antifungal therapy. A and B. CT scan demonstrates a solitary nodule of $<1 \mathrm{~cm}$ in diameter in the upper lobe of the left lung. 
of antifungal treatment was clear: the solitary nodule in the left lung disappeared and new lesions were not found. The skin lesion decreased in size and completely disappeared in 5 weeks. The patient continued to get chemotherapy and at present is in preparation to receive allogeneic haematopoietic stem cell transplantation from a matched unrelated donor.

\section{DISCUSSION}

Aspergillosis in immunocompromized patients is one of the most worrisome infectious complications. It is often described in patients with hemathological malignancies and in most cases presents as IA. Cutaneous aspergillosis, both primary and secondary to hematogenous dissemination of Aspergillus spp. is a relatively rare clinical manifestation.

An ulcerative necrotic skin lesion in a patient on chemotherapy is a frequent finding but usually a solitary, non-disturbing lesion without symptoms of generalized infection can be easily omitted. The place of fungal infection in such lesions is not dominant. A standard monitoring with Aspergillus galactomannan antigen test is useful in certain circumstances but its sensitivity varies between $52.9 \%$ and $79.3 \%$ (3) and cannot fully substitute the clinical examination.

PCA is an uncommon disease, mostly described in case reports (4-7). It typically develops on the place of disruption of skin integrity. This was not the case in the described patient, which makes it especially unusual for PCA.

Viewed in this way, a single small lung lesion detected on CT examination can be of special interest. This incidental finding is difficult to interpret. A solitary pulmonary nodule can be caused by a range of conditions varying from an infection or other benign lesion to a malignancy. Meanwhile, with improved imaging technique more nodules can be detected, but the explanation of the presence of solitary lesions remains unclear (8).

In neutropenic patients with leukemia the incidence of IA exceeds 4-5\% (9-11). The typical early findings in IPA are $\geq 1$ macronodules often with a "halo sign". Small solitary lesions $(<1 \mathrm{~cm})$ might be present in only a small percent of all patients with IPA (12). Indeed, if similar nodule is found on CT scan as part of a disseminated pulmonary process it would be taken as a part of the same infection, while just a solitary lesion is more likely to be interpreted as "non-specific".

In the present case, considering the proven skin aspergillosis, and disappearance of the lung lesion after specific therapy there is enough proof that both lesions belong to the same process and we have good reason to define the disease as IA.

After not finding any other focus of infection, the question which of these two loci is primary: the skin (proven) or the lung (probable) was raised.

We proved that the skin lesion was caused by A. flavus. A retrospective study on deep cutaneous fungal infections in immunocompromised children showed that Aspergillus spp. was the most common pathogen, responsible for $44 \%$ of the cases. In this study Aspergillus flavus was the most commonly isolated species (14).

Only relatively small conidia are able to evade the pulmonary clearance. A. fumigatus conidia are smaller in size than those of $A$. flavus. As a result A. fumigatus has a dominant role in IPA. Currently some data shows a growing frequency of other nonAspergillus fumigatus species (15-17).

In PCA cases, which develop due to direct fungal inoculation, the size of conidia does not play a significant role. But in addition to the size of the spores there are numerous factors, like surface characteristics, pigment composition, adhesion properties, resistance to phagocytosis, resistance to temperature and other environmental conditions, such as weather, etc., which influence the epidemiology, the way of clinical presentation e.g. preferable localization of primary invasion (14). This data demonstrates higher virulence of $A$. flavus than A. fumigatus. Therefore $A$. flavus, as a causative agent in our case, does not exclude primary invasion via lungs followed by rapid hematogenous dissemination to the skin which was the evident presentation in this patient. If a small skin lesion is missed, another, less favorable scenario could developed: disseminated bulky lung impairment and different end organ involvement, e.g. CNS. Such evolution would be more resistant to treatment and would have significantly worse prognosis.

Voriconazole belongs to the group of mold-active second generation azoles. It is approved for firstline treatment of IA and for cutaneous aspergillosis (18). Despite the fact that resistance to azoles in As- 
Milena Bozhkova, Temenuga Stoeva, Bonka Marinska et al.

pergillus spp. has already been reported, most studies show that the activity of voriconazole remains excellent $(19,20)$. In this case voriconazole demonstrated a full effect and the antileukemic program was not modified.

In conclusion, this "simple" case demonstrates that cutaneous aspergillosis may occur in immunosuppressed patients as part of probable invasive aspergillosis. The case shows that serial gallactomannan antigen evaluations do not substitute repeated lung CT scanning for monitoring for invasive fungal infection. The correct and rapid microbiological diagnosis and early start of specific treatment with proper antifungal agent is the key to a successful outcome in high-risk patients.

\section{REFERENCES}

1. Van Burik JA, Colven R, Spach DH. Cutaneous aspergillosis. J Clin Microbiol. 1998;36(11):3115-21.

2. Walsh TJ. Primary cutaneous aspergillosis-an emerging infection among immunocompromised patients. Clin Infect Dis . 1998;27(3):453-7.

3. Ascioglu S, Rex JH, De Pauw B, Bennett JE, Bille J, Crokaert F, et al. Defining opportunistic invasive fungal infections in immunocompromised patients with cancer and hematopoietic stem cell transplants: an international consensus. Clin Infect Dis. 2002, 34(1):7-14. doi: 10.1086/323335.

4. Dal T, Tekin A, Tekin R, Deveci Ö, Firat U, Mete $\mathrm{M}$, et al. Soft tissue abscess caused by Aspergillus fumigatus in an immunosuppressive patient. Eur J Gen Med. 2013;10:118-122.

5. Saghrouni F, Gheith S, Yaacoub A, Ammous O, Ben Abdeljelil J, Fathallah A, et al. Primary cutaneous aspergillosis due to Aspergillus flavus in a neutropenic patient. J Mycol Med. 2011; 21(4):285-8. doi: 10.1016/j.mycmed.2011.09.003.

6. Torrelo A, Hernández-Martín A, Scaglione,C Madero L, Colmenero I, Zambrano A. Primary cutaneous cspergillosis in a leukemic child. Actas Dermosifiliogr. 2007;98(4):276-8.

7. Darr-Foit S, Schliemann S, Scholl S, Hipler UC, Elsner P. Primary cutaneous aspergillosis - an uncommon opportunistic infection Review of the literature and case presentation. J Dtsch Dermatol Ges. 2017;15(8):839-41. doi: 10.1111/ddg.13297.

8. Wahidi MM, Govert JA, Goudar RK, Gould MK, McCrory DC, American College of Chest Physicians. Evidence for the treatment of patients with pulmonary nodules: when is it lung cancer?: ACCP evidence-based clinical practice guidelines (2nd edition). Chest. 2007;132(Suppl 3):94-107. doi: 10.1378/chest.07-1352.

9. Zaoutis TE, Heydon $\mathrm{K}, \mathrm{Chu} \mathrm{JH}$, Walsh TJ, Steinbach WJ. Epidemiology, outcomes, and costs of invasive aspergillosis in immunocompromised children in the United States, 2000. Pediatrics. 2006;117(4):711-16. doi: 10.1542/peds.2005-1161.

10. Crassard N, Hadden H, Piens MA, Pondarré C, Hadden R, Galambrun C, et al. Invasive aspergillosis in a paediatric haematology department: a 15-year review. Mycoses. 2008;51(2):109-16.doi: 10.1111/j.1439-0507.2007.01449.x.

11. Steinbach WJ. Invasive aspergillosis in pediatric patients. Curr Med Res Opin. 2010; 26(7):1779-87. doi: 10.1185/03007995.2010.487793.

12. Greene RE, Schlamm HT, Oestmann JW, Stark P, Durand C, Lortholary O, et al. Imaging findings in acute invasive pulmonary aspergillosis: clinical significance of the halo sign. Clin Infect Dis. 2007;44(3):373-9. doi: 10.1086/509917.

13. Marcoux D, Jafarian F, Joncas V, Buteau C, Kokta V, Moghrabi A. Deep cutaneous fungal infections in immunocompromised children. J Am Acad Dermatol. 2009;61(5):857-64. doi: 10.1016/j. jaad.2009.02.052.

14. Pasqualotto AC. Differences in pathogenicity and clinical syndromes due to Aspergillus fumigatus and Aspergillus flavus. Med Mycol. 2009;47(Suppl 1): 261-70. doi: 10.1080/13693780802247702.

15. Lass-Flörl C, Griff K, Mayr A, Petzer A, Gastl G, Bonatti $\mathrm{H}$, et al. Epidemiology and outcome of infections due to Aspergillus terreus: 10-year single centre experience. Br J Haematol. 2005;131(2):201-7. doi: 10.1111/j.1365-2141.2005.05763.x.

16. Mayr A, Lass-Flörl C. Epidemiology and antifungal resistance in invasive Aspergillosis according to primary disease: review of the literature. Eur J Med Res. 2011;16(4):153-7.

17. Marr KA, Carter RA, Crippa F, Wald A, Corey L. Epidemiology and outcome of mould infections in hematopoietic stem cell transplant recipients. Clin Infect Dis. 2002;34(7):909-17. doi: 10.1086/339202.

18. Patterson TF, Thompson GR 3rd, Denning DW, Fishman JA, Hadley S, Herbrecht R. Practice Guidelines for the Diagnosis and Management of Aspergillosis: 2016 Update by the Infec- 
tious Diseases Society of America. Clin Infect Dis. 2016;63(4):433-42. doi: 10.1093/cid/ciw444.

19. Snelders E, Van der Lee HA, Kuijpers J, Rijs AJ, Varga J, Samson RA, et al. Emergence of azole resistance in Aspergillus fumigatus and spread of a single resistance mechanism. PLoS Med. 2008;5(11):e219. doi: 10.1371/journal.pmed.0050219.

20. Koehler P, Hamprecht A, Bader O, BekeredjianDing I, Buchheidt D, Doelken G, et al . Epidemiology of invasive aspergillosis and azole resistance in patients with acute leukaemia: the SEPIA Study. Int J Antimicrob Agents. 2017;49(2):218-223. doi: 10.1016/j.ijantimicag.2016.10.019. 